\title{
Inactivation of Beef Brain $\alpha$-Ketoglutarate Dehydrogenase Complex by Valproic Acid and Valproic Acid Metabolites \\ Possible Mechanism of Anticonvulsant and Toxic Actions
}

\author{
Anthony S. Luder, ${ }^{\star}$ Janice K. Parks, ${ }^{\ddagger}$ F. Frerman, “ and William Davis Parker, Jr." \\ The B. F. Stolinsky Research Laboratories, Section of Genetics, ${ }^{*}$ Section of Pediatric Neurology, ${ }^{\S}$ Department of Pediatrics, and \\ Department of Neurology, ${ }^{\ddagger}$ University of Colorado School of Medicine, Denver, Colorado 80262
}

\begin{abstract}
The anticonvulsant valproic acid (VPA, 2-n-propylpentanoic acid) causes inhibition of the citric acid cycle and elevations of central nervous system (CNS) $\boldsymbol{\gamma}$-aminobutyric acid (GABA) levels, which correlates with anticonvulsant action. No unifying mechanism for these actions of VPA has won general acceptance. $\alpha$-Ketoglutarate dehydrogenase complex (KDHC) is a critical control enzyme in the CNS. We hypothesized that VPA may be an inhibitor of this enzyme since decreased KDHC activity would reduce substrate flux through the citric acid cycle and may increase flux into GABA synthesis. To test this hypothesis, inhibition of purified beef brain KDHC by VPA and its metabolites 2-n-propylpent-2-enoic acid $(\Delta 2,3$ VPE) and their coenzyme $A(C o A)$ derivatives were studied. Preincubation of the NADH-reduced enzyme with $\Delta 2,3$ VPE, VPA-CoA, and $\triangle 2,3$ VPE-CoA caused time-dependent inactivation, reversible by addition of CoA. Under steady-state conditions, $\triangle 2,3$ VPE and VPA-CoA were competitive inhibitors of KDHC and $\triangle 2,3$ VPE-CoA was a mixed inhibitor. These observations have implications for the molecular mechanisms of VPA action. VPA derivatives cause inactivation and inhibition of KDHC, which may explain the anticonvulsant and some toxic actions of VPA. (J. Clin. Invest. 1990 86:1574-1581.) Key words: valproic acid - Reye syndrome - gamma-aminobutyric acid $\bullet$ alpha-ketoglutarate dehydrogenase complex • anticonvulsant
\end{abstract}

\section{Introduction}

Valproic acid (VPA ${ }^{1}$; 2-n-propylpentanoic acid) is a branch chain fatty acid with anticonvulsant properties in humans and animals (1). VPA is an effective drug for a variety of seizure

Dr. Luder's present address is Department of Pediatrics, Carmell Hospital, Haifa, Israel.

Address reprint requests to Dr. Parker, Department of Neurology, Box C233, University of Colorado Health Sciences Center, 4200 East 9th Avenue, Denver, CO 80262.

Received for publication 10 March 1989 and in revised form 22 June 1990.

1. Abbreviations used in this paper: GABA, $\gamma$-aminobutyric acid; KDHC, $\alpha$-ketoglutarate dehydrogenase complex; MOPS, 3-[ $N$-morpholino]propane sulfonic acid; PDHC, pyruvate dehydrogenase complex; TPP, thiamine pyrophosphate; VPA, valproic acid; $\Delta 2,3$ VPE, $\Delta 2,3$ valproenic acid.

J. Clin. Invest.

(c) The American Society for Clinical Investigation, Inc. $0021-9738 / 90 / 11 / 1574 / 08 \quad \$ 2.00$

Volume 86, November 1990, 1574-1581 disorders, but its use is limited by its toxicity, which may be severe or occasionally fatal (2). Better understanding of the mechanisms of anticonvulsant and toxic actions of this drug might enable them to be separated and improved drugs designed. Since the biochemical toxicity of VPA resembles that seen in Reye's syndrome and certain inborn errors of metabolism, better understanding of VPA biochemistry might also provide some general insights into the mitochondrial dysfunction common to these conditions.

The anticonvulsant action of VPA is correlated with increased $\gamma$-aminobutyric acid (GABA) levels in the central nervous system (CNS) of VPA-treated rats (3). The mechanism of this GABA-elevating action is incompletely understood (4). VPA administration leads to increased incorporation of ${ }^{14} \mathrm{C}$ labeled glucose into GABA via the citric acid cycle (5) but the enzymatic site at which this occurs is not known. A clue to the possible site of action comes from the observations of that VPA is also an inhibitor of the mitochondrial citric acid cycle (6-9). A candidate single site for both of these VPA actions is $\alpha$-ketoglutarate dehydrogenase complex (KDHC) (EC 1.2.4.2, 2.3.1.61, 1.6.4.3). The activity of this multienzyme complex is probably rate-limiting for citric acid cycle activity in CNS (10) and its substrate, $\alpha$-ketoglutarate, can be utilized directly for GABA synthesis through glutamate (11). We hypothesized that VPA or VPA derivatives may be inhibitors of KDHC, since KDHC activity would lead to the observed inhibition of the citric acid cycle and could account for increased shunting of carbon skeletons into glutamate and GABA. Previously published data showing that oxygen utilization by rat liver mitochondria is inhibited by VPA if glutamate is a substrate (12), but not if GABA or succinate are substrates (13), suggests that the locus of VPA-mediated inhibition of the citric acid cycle is proximal to succinyl-CoA $(12,13)$ and is also consistent with our hypothesis.

In order to test the proposed inhibition of $\mathrm{KDHC}$, we decided to examine VPA and pertinent metabolites in kinetic studies with the purified beef brain enzyme. Because there is evidence that tissue isoenzymes of KDHC may exist (14), we chose to study the brain enzyme, although KDHC from other tissues has been extensively characterized (15). A new method for purifying this enzyme was required because $\mathrm{KDHC}$ is present in relatively low concentration in the CNS (10) and the high lipid content of brain may impair its stability. Inhibition studies were performed with VPA and its major monounsaturated metabolite $\Delta 2,3$ valproenic acid (2- $n$-propylpent-2-enoic acid, $\Delta 2,3$ VPE) and their coenzyme $A(C o A)$ esters. Ito et al. (16) have shown that VPA-CoA, which is present in rat liver during administration of VPA, is oxidized to $\triangle 2,3$ VPE-CoA by $\alpha$-methylbutyryl-CoA dehydrogenase. $\Delta 2,3$ VPE was studied because anticonvulsant activity is better correlated with its plasma and tissue levels than the parent molecule $(17,18)$. 


\section{Methods}

Reagents. All reagents were of analytic grade. Sepharose CL-2B and agarose-NAD ${ }^{+}$(AgNAD type 3) were obtained from Pharmacia Inc., Piscataway, NJ. Bio-Beads were obtained from Bio-Rad Laboratories, Richmond, CA. VPA and $\triangle 2,3$ VPE were supplied by Saber Laboratories, Morton Grove, IL. Tetrahydrofuran and triethylamine were obtained from Fisher Scientific Co., Fair Lawn, NJ. Ethyl chloroformate was supplied by Aldrich Chemical Co., Milwaukee, WI. Acetonitrile was obtained from J. T. Baker Chemical Co., Phillipsburg, NJ. All other reagents were supplied by Sigma Chemical Co., St. Louis, MO.

Enzyme assays. KDHC was assayed according to the method of Jackson and Singer (19). Pyruvate dehydrogenase complex (PDHC) was assayed by the same method except that sodium pyruvate $(4 \mathrm{mM})$ was substituted for alpha-ketoglutarate. Maximum activity of KDHC and PDHC when assayed in crude tissue was obtained by presolubilization for $5 \mathrm{~min}$ with $0.1 \%$ Luberol (WX) and addition of $2 \mu \mathrm{M}$ rotenone to inhibit NADH oxidoreductase activity. A diode array spectrophotometer (model 8452, Hewlett-Packard Co., Palo Alto, CA) was used to follow the reduction of $\mathrm{NAD}^{+}$at $340 \mathrm{~nm}$, and $\mathrm{Fe}(\mathrm{CN})_{6}^{-3}$ at $420 \mathrm{~nm}$. Specific enzyme activity is expressed as micromoles of $\mathrm{NAD}^{+}$ reduced per minute per milligram of protein at $25^{\circ} \mathrm{C}$. Kinetic constants and standard errors were calculated according to Wilkinson (20).

Protein was determined by the method of Lowry et al. (21). Sodium dodecyl sulphate (SDS) $0.1 \%$ (3.47 mM) was added to prevent interference from Triton X-100 which forms a precipitate with the Folin reagent.

Gels. Analytical SDS/polyacrylamide gel electrophoresis was carried out as described by Laemmli (22) on 10-13\% gradient gels. Samples of protein for loading onto gels were prepared as described by Perham and Thomas (23).

Fast-atom bombardment mass-spectrophotometry. Fast-atom bombardment mass-spectrometry of CoA esters was carried out on aqueous solutions with a mass spectrometer (model 7070E, V. G. Analytical, Manchester, UK).

Statistical analysis and reproducibility. Statistical evaluation was carried out using the Crunch statistical program (Crunch Software, Oakland, CA). Information concerning reproducibility is contained in the legends of tables and figures. Kinetic constants are calculated from the results of three experiments, done in duplicate, in each case.

Preparation of $K D H C$. All procedures were carried out at $4^{\circ} \mathrm{C}$ unless otherwise stated. Buffer $\mathrm{pH}$ values were adjusted at $20^{\circ} \mathrm{C}$.

Preparation of beef brain mitochondria. Two beef brains were chilled immediately after slaughter and trimming. The brains were diced in chilled isotonic buffer $(50 \mathrm{mM}$ Tris, $0.25 \mathrm{M}$ sucrose, $2.7 \mathrm{mM}$ EDTA, pH 7.4) and homogenized by six passes in a 500-ml glass-teflon homogenizer. The final volume was about 1.5 liter. The homogenate was centrifuged for $5 \mathrm{~min}$ at $3,000 \mathrm{~g}$ and the supernatant was retained. The pellet was rehomogenized and centrifuged as above. The combined supernatants were centrifuged at $17,000 \mathrm{~g}$ for $10 \mathrm{~min}$. To rupture synaptosomes, the pellet was suspended in hypotonic buffer containing $6 \mathrm{mM}$ Tris, $2.7 \mathrm{mM}$ EDTA, $\mathrm{pH} 7.4$, and quickly hand homogenized in a final volume of $250 \mathrm{ml}$. The preparation was centrifuged for $10 \mathrm{~min}$ at $17,000 \mathrm{~g}$, the supernatant was discarded, and this step was repeated on the pellet. To remove excess lipid, the sedimented mitochondria were taken up in $100 \mathrm{ml}$ of the isotonic buffer and carefully layered in aliquots on Ficoll $7 \%$ (wt/wt, in isotonic buffer). The aliquots were centrifuged at $11,500 \mathrm{~g}$ for $30 \mathrm{~min}$. The resulting pellet, which now had a pronounced brown color, was used as the source of mitochondria.

Brain mitochondria were taken up in $400 \mathrm{ml}$ of buffer containing $50 \mathrm{mM}$ 3-[ $N$-morpholino]propanesulfonic acid (MOPS), 3\% Triton $\mathrm{X}-100$ (vol/vol), $2.7 \mathrm{mM}$ EDTA, $1 \mathrm{mM}$ benzamidine, $1 \mathrm{mM}$ PMSF, $0.1 \mathrm{mM}$ thiamine pyrophosphate (TPP), $0.1 \mathrm{mM}$ DTT, $\mathrm{pH}$ 7.4. The suspension was stirred for $30 \mathrm{~min}$ and centrifuged at $25,000 \mathrm{~g}$ for 10 $\mathrm{min}$. The golden supernatant was brought to $10 \mathrm{mM} \mathrm{Mg}^{2+}$ by the slow addition of $\mathrm{MgCl}_{2}$ from a $1 \mathrm{M}$ stock solution and the $\mathrm{pH}$ reduced to 6.45 by dropwise addition of $10 \%$ acetic acid. Polyethylene glycol (PEG) 35\% was quickly added from a 35\% stock solution to a final concentration of $4.2 \%$ and the suspension stirred for $30 \mathrm{~min}$. The suspension was centrifuged at $25,000 \mathrm{~g}$ for $40 \mathrm{~min}$ and the supernatant ( $<5 \%$ total KDHC activity) was discarded. The pellet was resuspended in $5-9 \mathrm{ml}$ of buffer containing $50 \mathrm{mM}$ potassium phosphate, $1 \mathrm{mM}$ benzamidine, $1 \mathrm{mM}$ PMSF, $0.1 \mathrm{mM}$ DTT, $0.1 \mathrm{mM}$ TPP, $2.7 \mathrm{mM}$ EDTA, pH 7.4, and sonicated for $30 \mathrm{~s}$ at $40 \mathrm{~W}$ with a sonicator (model W-225, Heat Systems-Ultrasonics, Farmingdale, NY) to resuspend. To reduce micelle formation by residual lipid, Triton was removed by passing the solution over a $1 \times 6-\mathrm{cm}$ column of Bio-Beads, equilibrated with the same buffer, under gravity. KDHC was partially purified by gel filtration on a $2.5 \times 90-\mathrm{cm}$ Sepharose CL-2B column, using the same phosphate buffer without Triton. Fractions containing highest specific KDHC activity were pooled and volume reduced to $10-20 \mathrm{ml}$ by ultrafiltration using an Amicon PM 30 membrane (W. R. Grace \& Co. Danvers, MA). Elution profiles from repeated preparations were very similar. A typical elution profile is shown in Fig. 1.

Affinity chromatography was used to further purify KDHC. The preparation was made $10 \mathrm{mM}$ with respect to $\mathrm{Mg}^{2+}$ by dropwise addition of $1 \mathrm{M} \mathrm{MgCl}_{2}$ and was made $0.1 \mathrm{mM}$ with respect to $\alpha$-ketoglutarate by addition of its potassium salt. $\mathrm{pH}$ was maintained at 7.4. The preparation was loaded by gravity onto a preequilibrated $1 \times 3-\mathrm{cm}$ column of AgNAD type 3, equilibrated with the same buffer. After washing with 10 volumes of the buffer to constant absorbance at 280 $\mathrm{nm}, \mathrm{KDHC}$ was eluted using a linear gradient of $\mathrm{NAD}^{+}(0.5-2 \mathrm{mM})$ in loading buffer without $\alpha$-ketoglutarate (total volume $80 \mathrm{ml}$, at a rate of $1 \mathrm{ml} / \mathrm{min}$ ). All detectable PDHC activity was removed in the initial wash. Fractions of $1 \mathrm{ml}$ were collected. Fractions 7-25 contained 95\% of detectable KDHC activity in three different experiments. These fractions were pooled and ultrafiltered to a concentration of 50-100 $\mu \mathrm{g} / \mathrm{ml}$ on an Amicon PM 30 membrane, and stored at $-22^{\circ} \mathrm{C}$ in $50 \%$ glycerol. Activity was stable in these conditions for at least $12 \mathrm{wk}$.

The purification procedure is summarized in Table I. SDS/polyacrylamide gel electrophoresis demonstrated three bands of apparent molecular weights of 113,000 $\left(E_{1}\right), 61,000\left(E_{3}\right)$ and 54,000 $\left(E_{2}\right)$ (Fig. 2). $E_{1}$ corresponds closely to published values from other tissues (15) although apparent molecular weights for $E_{2}$ and $E_{3}$ in other species vary slightly (24-26).

Synthesis of VPA and $\triangle 2,3$ VPE-COA. The CoA esters of VPA and $\triangle 2,3$ VPE were synthesized via the mixed anhydride method, as described by Bernert and Sprecher (27) using distilled tetrahydrofuran as the solvent for preparation of the anhydrides. CoA esters were partially purified by filtration on a DEAE-cellulose column using a gradient of $\mathrm{LiCl}(0.01-0.25 \mathrm{M})$ in $6 \mathrm{mM} \mathrm{HCl}$ to elute. After desalting on a 2 $\times 45-\mathrm{cm}$ Sephadex G-10 column and eluting with water, the product was adsorbed onto a $1.1 \times 1.3-\mathrm{cm} \mathrm{C18} \mathrm{Sep-pak} \mathrm{cartridge} \mathrm{(Waters}$ Associates, Milford, MA), previously equilibrated successively with

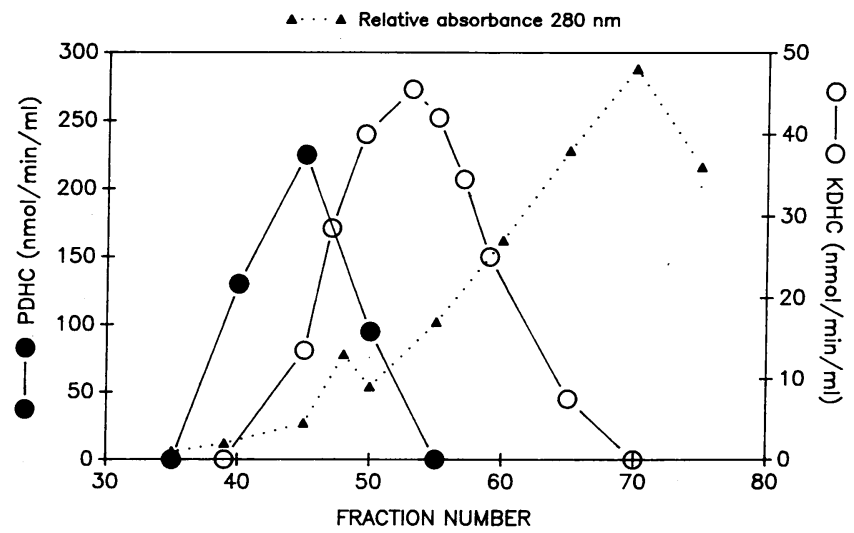

Figure 1. Gel filtration of partly purified $\alpha$-KDHC from beef brain on Sepharose CL-2B. The buffer was $50 \mathrm{mM}$ potassium phosphate, 1 $\mathrm{mM}$ benzamidine, $1 \mathrm{mM}$ PMSF, $0.1 \mathrm{mM}$ DTT, $0.1 \mathrm{mM}$ TPP, 2.7 mM EDTA, pH 7.4. See text for further experimental details. 
Table I. Purification of $\alpha-K D H C$ from Beef Brain

\begin{tabular}{lccccc}
\hline \multicolumn{1}{c}{ Step } & Final volume & Protein & Enzyme activity & Specific activity & Yield \\
\hline & $m l$ & $m g$ & $n$ mol/min & mmol/mg per min & $\%$ \\
Crude Triton extract & 400 & 4854 & 52,190 & 0.0109 & $100^{*}$ \\
Poly(ethylene glycol) precipitation & 9 & 1320 & 52,000 & 0.0394 & 99 \\
Gel filtration on Sepharose CL-2B & 20 & 376 & 16,930 & 0.442 & 32 \\
Affinity gel filtration on AgNAD type 3 & 20 & 3.9 & 13,756 & 3.5 & 26
\end{tabular}

All values refer to a preparation from about $350 \mathrm{~g}$ of beef brain. ${ }^{*}$ Accurate values for $\alpha$-KDHC were difficult to obtain before Triton extraction because of NADH oxidases.

HPLC-grade acetonitrile, water, and $50 \mathrm{mM}$ ammonium acetate, $\mathrm{pH}$ 5.3. The product was eluted with a discontinuous gradient of $15 \%$ acetonitrile in $50 \mathrm{mM}$ ammonium acetate (pH 5.3) followed by $30 \%$ acetonitrile in $50 \mathrm{mM}$ ammonium acetate. The material eluted in the $30 \%$ fraction was lyophilized and stored at $-22^{\circ} \mathrm{C}$.

Identification and purity of $\mathrm{CoA}$ esters were assessed as follows. HPLC using the methanol/phosphate/acetonitrile buffer gradient as described by Causey et al. (28) revealed a single peak. FAB-MS showed major ion peaks at 894 and 892 for VPA-CoA and $\triangle 2,3$ VPE-CoA, respectively. These correspond to the calculated molecular weights of these two compounds. Absorption spectra showed a ratio at 232 $\mathrm{nm} / 260 \mathrm{~nm}$ of 0.42 for VPA-CoA and 0.56 for $\Delta 2,3$ VPE-CoA (Fig. 3). Thin-layer chromatography on cellulose thin layer (Eastman Kodak 13181, Rochester, NY) by the method of Myers and Utter (29) showed single spots which migrated slightly ahead of a tiglyl-CoA standard when visualized by UV light. After alkaline hydrolysis, these spots disappeared and new spots running with CoA standard appeared.

\section{Results}

Enzymatic properties of beef brain KDHC. Cofactor dependency and substrate specificity were determined for beef brain $\mathrm{KDHC}$. Under steady-state conditions, assays of $\mathrm{NAD}^{+}$reduction showed marked substrate specificity. The following substrates were tested at $4 \mathrm{mM}$ (the order of magnitude seen in inherited metabolic disease): pyruvate, $\alpha$-keto-3-methylvalerate, $\alpha$-ketoisocaproate, $\alpha$-ketobutyrate, and oxaloacetate. VPA and $\Delta 2,3$ VPE were tested at $20 \mathrm{mM}$, concentrations possible during therapy or overdose. No activity was seen with any of these substrates. Compared with $\alpha$-ketoglutarate $(=100 \%$ activity), activity with $\alpha$-ketoisovalerate was $1.7 \%$, with acetoacetate $2.3 \%$, and $16.6 \%$ with $\alpha$-ketoadipate (all tested at $4 \mathrm{mM}$ ). These results are similar to those reported with KDHC from pig heart (30). It should be noted that KDHC in high concen-

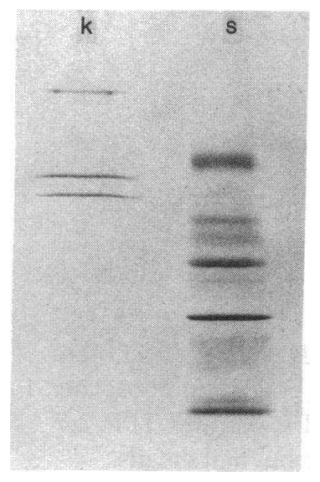

Figure 2. SDS/polyacrylamide gel $(10-13 \% \mathrm{wt} / \mathrm{vol})$ electrophoresis of $\alpha$ KDHC from beef brain $(K)$ and a standard polypeptide mixture $(S)$ (SDS 7, Sigma Chemical Co.). tration isolated from beef heart may show extremely low rates of $\mathrm{NAD}^{+}$reduction when branch-chain amino acids are substrates $(0.04-0.15 \%$ compared to $\alpha$-ketoglutarate [19]) but that such activity was not detected with KDHC from pig heart (30, 31). Dehydrogenase activity showed partial dependency on cations. At saturating substrate concentrations, total activity was $28 \%$ of maximum in the absence of $\mathrm{Ca}^{2+}$ or $\mathrm{Mg}^{2+}$, both of which were effective activators. EDTA did not further reduce activity in the absence of cations. $\mathrm{Mn}^{2+}$ and $\mathrm{Ba}^{2+}$ were also effective activators but $\mathrm{Zn}^{2+}$ and $\mathrm{Cu}^{2+}$ irreversibly inactivated the enzyme. Exogenous TPP was required for full activity but $70 \%$ activity was seen in its absence, presumably owing to enzyme bound TPP.

The steady-state kinetics of KDHC are complex (32-34); substrate concentrations less than $10 \times K_{\mathrm{m}}$ can substantially reduce the apparent $V_{\max }$. We chose to examine some kinetic
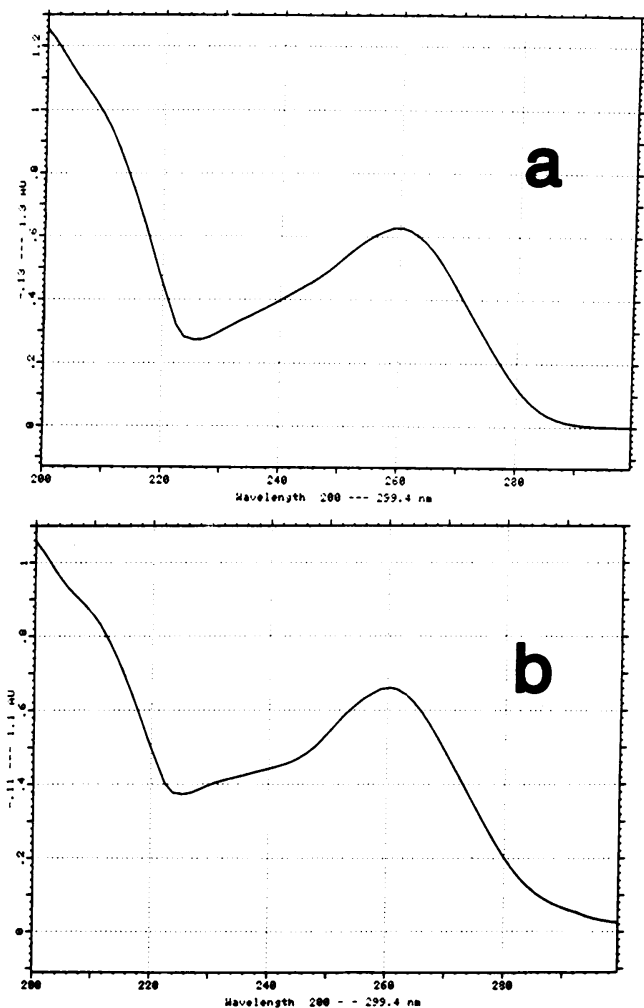

Figure 3. (a) Absorption spectra of VPA-CoA and (b) $\delta 2,3$ VPE-CoA 200-300 nm. Spectra were recorded on a diode array spectrophotometer (model 990, Waters Associates). 
constants of brain KDHC for its physiologic substrates. For each constant, one substrate was tested at varying concentrations and other substrates were at saturating concentrations $\left(100 \times K_{\mathrm{m}}\right)$. Kinetic constants for CoA and $\alpha$-ketoglutarate and inhibition by succinyl CoA with varied concentrations of CoA are shown in Table II. In these conditions, the enzymatic properties of the preparation from beef brain are similar to those previously reported in other tissues $(19,34-37)$.

Inactivation of $K D H C$ by VPA metabolites. Preincubation of $\mathrm{KDHC}(0.1 \mathrm{mg} / \mathrm{ml})$ with $2 \mathrm{mM} \Delta 2,3 \mathrm{VPE}$ at $25^{\circ} \mathrm{C}$ caused significant time-dependent loss of catalytic activity $(P<0.005)$ (Fig. 4), but VPA up to $20 \mathrm{mM}$ showed no inactivation. 0.1 $\mathrm{mg} / \mathrm{ml} \mathrm{KDHC}$ was used since more dilute solutions were less stable and activity too low for accurate assay. Inhibitor concentrations were 10-100-fold their steady-state $K_{\mathrm{m}}$ to ensure saturating conditions. Attempts to reactivate the enzyme by extensive dialysis at $4^{\circ} \mathrm{C}$ failed, whereas control activity was unaffected by dialysis. Preincubation of NADH-reduced $\mathrm{KDHC}$ with both VPA-CoA and $\triangle 2,3$ VPE-CoA caused similar significant inactivation when incubated for up to $120 \mathrm{~min}$ $(P<0.001)$ (Fig. 5), although steady-state kinetic studies showed different patterns of inhibition (see below). "Rescue" experiments were performed, in which enzyme inactivated by $\Delta 2,3$ VPE-CoA as described above was further incubated with either CoA, $\mathrm{NAD}^{+}$, or $\alpha$-ketoglutarate. $95-100 \%$ activity was restored by treatment with $\mathrm{CoA}$ (Fig. 5), but not with $\mathrm{NAD}^{+}$or $\alpha$-ketoglutarate (data not shown).

Steady-state kinetics of KDHC inhibition. Initial velocity assays of $\mathrm{KDHC}$ were done at different concentrations of VPA and $\triangle 2,3$ VPE at saturating $\mathrm{NAD}^{+}$and $\mathrm{CoA}$ concentrations. Reactions were initiated with enzyme to accurately estimate initial rates, before inactivation of enzyme occurred. Assay of $\mathrm{E}_{1}$ subunit-mediated reduction of $\mathrm{Fe}(\mathrm{CN})_{6}^{-3}$ showed no inhibition by VPA or $\Delta 2,3$ VPE. In contrast, $\Delta 2,3$ VPE inhibited the reduction of $\mathrm{NAD}^{+}$by $\mathrm{KDHC}$ and was a competitive inhibitor with respect to $\alpha$-ketoglutarate (Fig. $6 \mathrm{~A}$ ). Under these conditions, $K_{\text {is }}$ was $41 \pm 1.3 \mu \mathrm{M}$. The Hill coefficient (Fig. $6 B$ ) was

Table II. Some Kinetic Constants for $\alpha-K D H C$ from Beef Brain and Other Tissues

\begin{tabular}{|c|c|c|}
\hline Substrate & $K_{\mathrm{m}}$ & Reported $K_{\mathrm{m}}$ (tissue) \\
\hline & $\mu M$ & $\mu M$ \\
\hline \multirow[t]{3}{*}{$\alpha$-Ketoglutarate } & $113 \pm 19$ & 110 (pig heart) (34) \\
\hline & & 13 (pig heart) (35) \\
\hline & & 81 (bovine liver mitochondria) (19) \\
\hline \multirow[t]{3}{*}{ CoA } & $1.3 \pm 0.2$ & 4.5 (pig heart) $(36)$ \\
\hline & & $<0.1$ (pig heart) $(35)$ \\
\hline & & 2.7 (rat liver) (32) \\
\hline \multirow[t]{2}{*}{ Inhibitor } & $K_{\text {is }}$ & \\
\hline & $\mu M$ & \\
\hline Succinyl CoA & $3.9 \pm 0.4$ & 6.9 (rat liver) (32) \\
\hline
\end{tabular}

Assays were performed by initiating the reaction with the test substrate in the presence of saturating concentrations $\left(10-100 \times K_{\mathrm{m}}\right)$ of other substrates. Inhibition by succinyl-CoA was studied under steady-state conditions in saturating concentrations of $\mathrm{NAD}^{+}$and $\alpha$ ketoglutarate. Results were calculated from the data collected from three repeat experiments in each case.

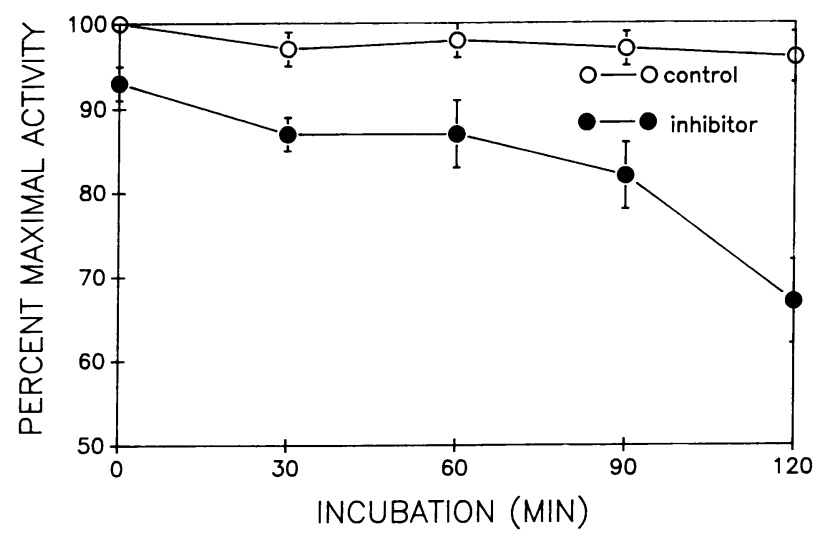

Figure 4. Inactivation of purified beef brain $\alpha-\mathrm{KDHC}$ by $\delta 2,3 \mathrm{VPE}$. The complex $(0.1 \mathrm{mg} / \mathrm{ml})$ was incubated at $25^{\circ} \mathrm{C}$ in $50 \mathrm{mM}$ MOPS, $2 \mathrm{mM} \mathrm{MgCl}_{2}, 0.5 \mathrm{mM} \mathrm{CaCl}_{2}, 2.6 \mathrm{mM}$ cysteine, $0.1 \mathrm{mM}$ TPP, pH 7.6, either in the presence of $2 \mathrm{mM} \delta 2,3 \mathrm{VPE}$ (inhibitor) or not (control). Aliquots taken at intervals were assayed as described in the text. Each data point represents the mean of duplicate determinations from three experiments, with error bars indicating 1 SD. The ordinate represents percentage of initial rate activity of an untreated sample. At $120 \mathrm{~min}$, activity in the preincubated samples was signifi- . cantly lower than control $(P<0.005)$.

0.5 , suggesting negative cooperativity and half-maximal inhibition occurred at $2.04 \mathrm{mM}$ (intercept at $\log \left[V_{\mathrm{o}} /\left(V_{\mathrm{i}}-V_{\mathrm{o}}\right)\right]$ $=0$ ). VPA did not inhibit at concentrations as high as $10 \mathrm{mM}$.

VPA-CoA and $\Delta 2,3$ VPE-CoA were both inhibitors of brain KDHC under steady-state conditions. VPA-CoA was a competitive inhibitor with respect to CoA with an apparent $K_{\text {is }}$ of $2.9 \pm 0.2 \mu \mathrm{M}$ (Fig. $7 A$ ). $\Delta 2,3$ VPE-CoA showed characteris-

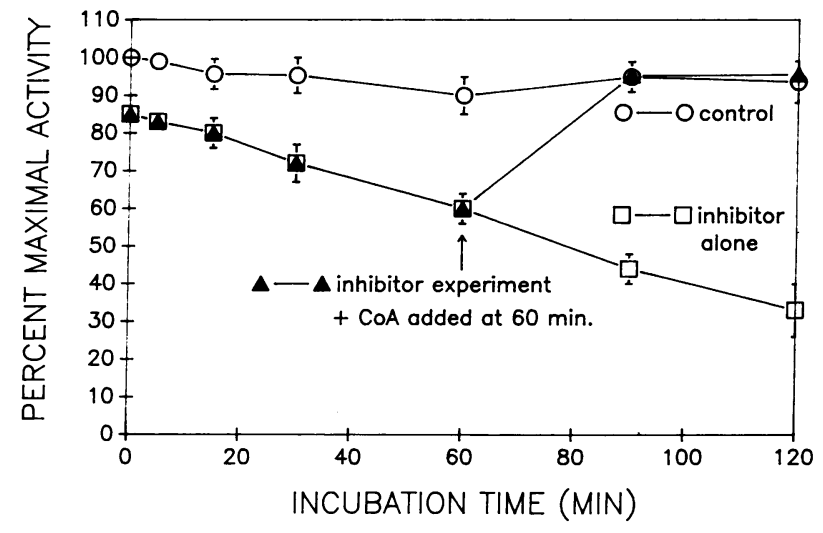

Figure 5. Inactivation of purified beef brain $\alpha-\mathrm{KDHC}$ by $\delta 2,3$ VPECoA. The complex $(0.1 \mathrm{mg} / \mathrm{ml})$ was incubated at $25^{\circ} \mathrm{C}$ in $50 \mathrm{mM}$ MOPS, $2 \mathrm{mM} \mathrm{MgCl}, 0.5 \mathrm{mM} \mathrm{CaCl}_{2}, 0.1 \mathrm{mM}$ cysteine, $0.1 \mathrm{mM}$ TPP, pH 7.6, either in the presence of $1 \mathrm{mM} \delta 2,3 \mathrm{VPE}-\mathrm{CoA}$ (inhibitor) and $1 \mathrm{mM} \mathrm{NADH}$ or in $1 \mathrm{mM} \mathrm{NADH}$ only (control). Aliquots were taken at intervals and assayed as described in the text. Each data point represents the mean of duplicate determinations from three experiments, with error bars indicating 1 SD. The ordinate represents percentage of initial rate activity of an untreated sample. Activity at 90 and $120 \mathrm{~min}$ was significantly lower in the preincubated samples $(P<0.0190 \mathrm{~min}, P<0.001120 \mathrm{~min})$. No significant difference in activity from control if $0.5 \mathrm{mM} \mathrm{CoA}$ was added to aliquots at $60 \mathrm{~min}$ and assayed at 90 and $120 \mathrm{~min}$. Incubation with VPACoA gave similar results. 

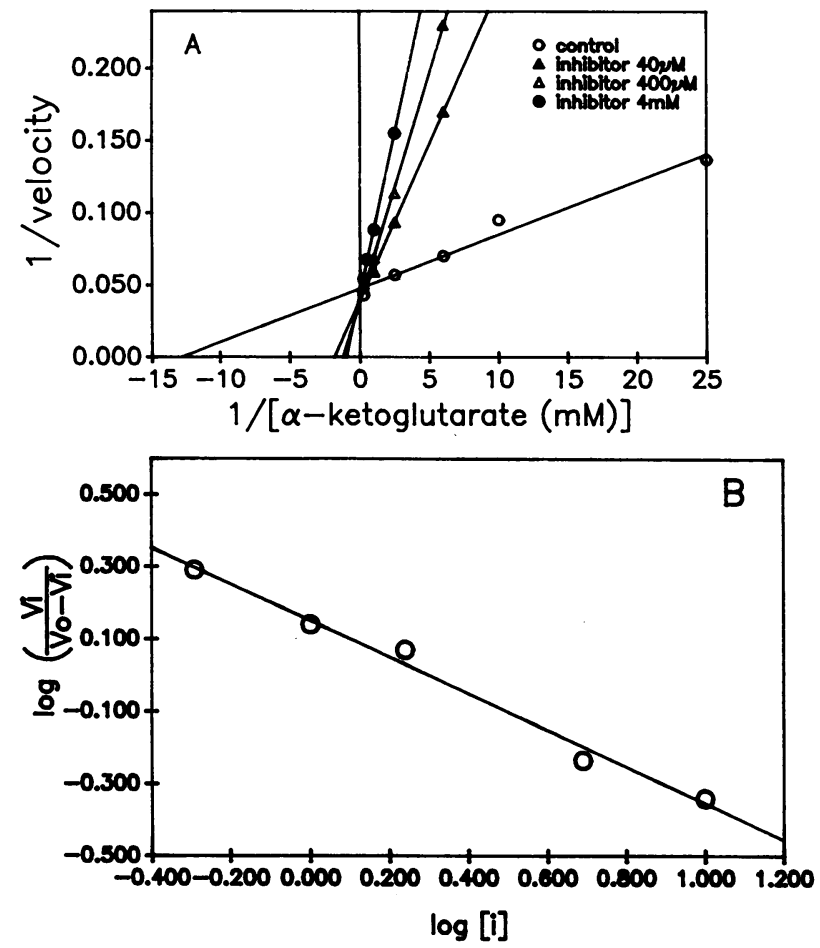

Figure 6. $(A)$ Inhibition of $\mathrm{NAD}^{+}$reduction by purified beef brain $\alpha$ $\mathrm{KDHC}$ with $\delta 2,3 \mathrm{VPE}$. Inhibitor was added to the assay buffer with $\alpha$-ketoglutarate to initiate the reaction. For other assay conditions see details in text. (B) Inhibition of brain $\alpha-\mathrm{KDHC}$ presented as a Hill plot. $V_{\mathrm{i}}=$ initial velocity in the presence of inhibitor, $V_{\mathrm{o}}=$ initial velocity under the same conditions in the absence of inhibitor. $\alpha$-Ketoglutarate concentration was $113 \mu \mathrm{M}\left(=K_{\mathrm{m}}\right)$. Other substrate concentrations as described in the text.

tics of a mixed type inhibitor (Fig. $7 B$ ). $K_{\mathrm{ii}}$ was $1.0 \pm 0.03 \mu \mathrm{M}$ and $K_{\text {is }}$ was $6.1 \pm 0.4 \mu \mathrm{M}$. These experiments were repeated with variable enzyme concentrations at fixed concentrations of CoA at its $K_{m}(1.3 \mu \mathrm{M})$. The results are shown in Fig. 8. The pattern of inhibition shown by VPA-CoA was typical of a competitive inhibitor and that of $\triangle 2,3$ VPE-CoA of mixed type inhibition. Hill plots of data from the inhibition of KDHC by $\Delta 2,3$ VPE-CoA gave a Hill coefficient of 0.74 suggesting negative cooperativity. Half-maximal inhibition concentration was $1.39 \mu \mathrm{M}$ (Fig. 9).

The metabolic effects of VPA in vivo resemble those seen in some inherited disorders of metabolism. Experiments were therefore performed to compare kinetic properties of some naturally occurring keto-acyl CoAs with those of the VPA metabolites. These experiments are summarized in Table III. The esters examined all showed marked inhibition of KDHC. All showed characteristics of competitive inhibitors with respect to CoA, except isobutyryl-CoA which was a mixed inhibitor (Fig. 10) and showed kinetic properties similar to those of $\triangle 2,3$ VPE-CoA. Isobutyryl-CoA is known to be a suicide inhibitor which inactivates $\mathrm{KDHC}$, presumably owing to covalent modification of the $E_{2}$ subunit (19).

\section{Discussion}

The results reported here are consistent with a proposed mechanism of VPA action in which inhibition and/or inactivation of KDHC by VPA would reduce citric acid cycle flux and increase flux into GABA synthesis. We have demonstrated that KDHC from mammalian brain is inactivated and inhibited by VPA metabolites. Chronic administration of VPA in therapeutic doses in dogs produced brain tissue levels of $\Delta 2,3$ VPE of $\sim 0.5-2 \mu \mathrm{M}$ and VPA was considerably higher (38). Furthermore, VPA and its metabolites have been shown to accumulate 10-fold in mitochondria (39). Although data describing steady-state intramitochondrial concentrations of CoA metabolites of VPA are lacking, even a conservative estimate based upon this published data suggests that they are present in concentrations well above the measured apparent $K_{\mathrm{i}}$ values as determined by us. In addition, inactivation of KDHC by unsaturated VPA derivatives could provide a theoretic basis for the prolonged action of VPA (40-42) and be consistent with correlation of clinical effect with serum levels of unsaturated metabolites. During therapy, inactivation of enzyme and its resynthesis or reactivation would be in a steady state, at a lower KDHC concentration than before treatment. After discontinuation of therapy, previous $\mathrm{KDHC}$ levels would only return after new synthesis and clearing of protein bound inhibitor. A precedent for inactivation by a similar molecule is isobutyryl-CoA, which is a known suicide inhibitor of $\mathrm{KDHC}$ (19). This compound is elevated in disorders of propionate and methylmalonic acid metabolism, conditions whose clinical and biochemical complications are very similar to VPA toxicity.

The results reported here demonstrating inactivation of KDHC by VPA metabolites have implications for the molecular mechanism of such inactivation. $\triangle 2,3$ VPE inactivated $\mathrm{KDHC}$ whereas the saturated parent compound VPA did not.

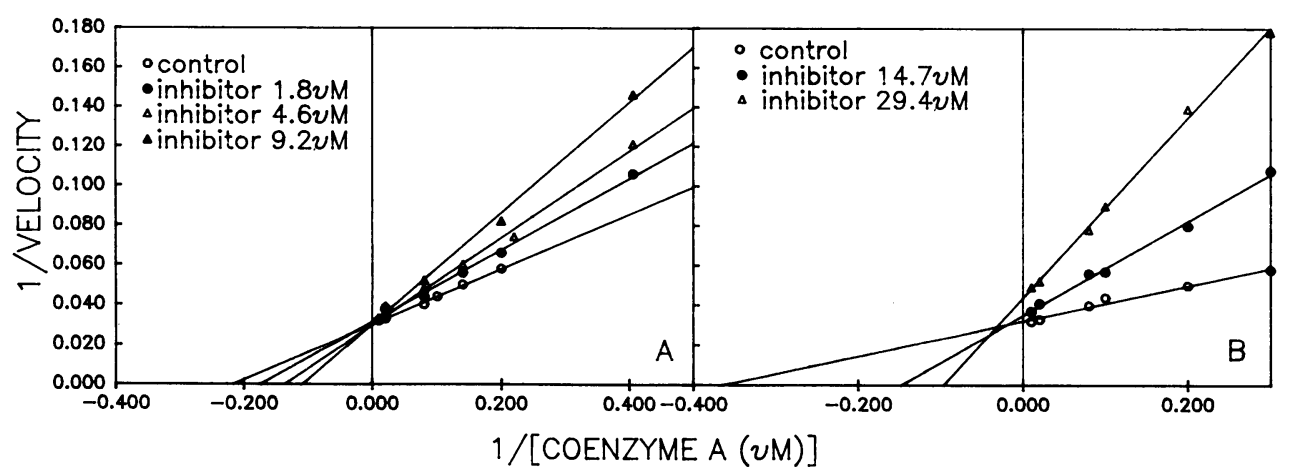

Figure 7. Inhibition of purified beef brain $\alpha$-KDHC by $(A)$ VPACoA and $(B) \delta 2,3$ VPE-CoA. Inhibitor was added with CoA to the assay buffer to initiate the reaction. For other assay conditions see details in text. 


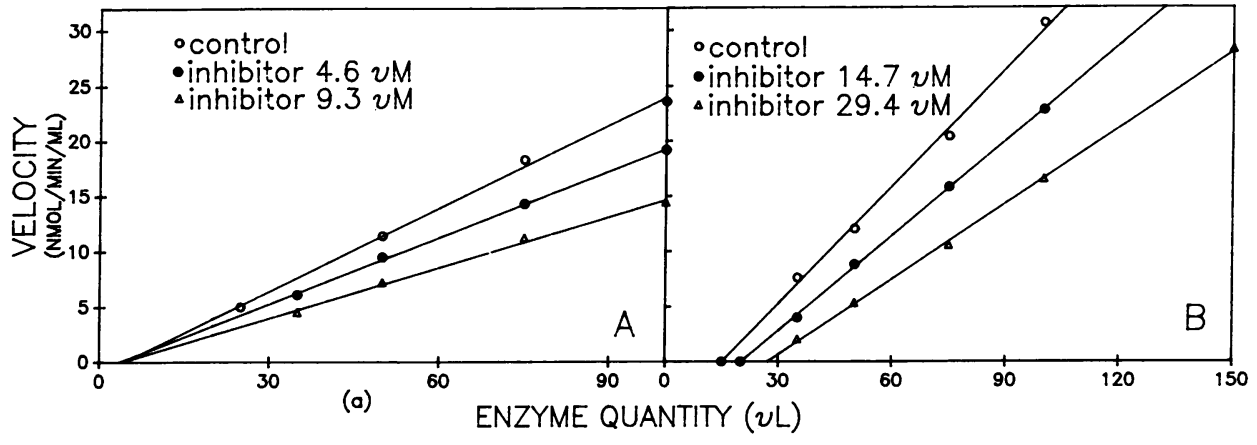

Figure 8. Variable enzyme plots of inhibition of purified beef brain $\alpha$ $\mathrm{KDHC}$ by $(A)$ VPA-CoA $(5 \mu \mathrm{M}=2$ $\left.\times K_{\text {ii }}\right)$ and $(B) \delta 2,3$ VPE-CoA $(5$ $\left.\mu \mathrm{M}=5 \times K_{\mathrm{ii}}\right)$. Assays were initiated by the addition of $\alpha$-ketoglutarate and inhibitor to the assay buffer. CoA concentration was 1.3 $\mu \mathrm{M}\left(=K_{\mathrm{m}}\right)$. Assays were carried out with increasing volumes of enzyme $(0.1 \mathrm{mg} / \mathrm{ml})$. For details of assay conditions see text.
Since it is known that double bonds in $\Delta 2,3$ unsaturated acids are susceptible to nucleophilic attack (for example, during the chemical synthesis of unsaturated fatty acyl-CoA esters [27]), it is possible that such a mechanism could explain the difference in behavior of the two molecules, although alternative possibilities such as allosteric effects are not excluded. Tight, probably covalent binding to the enzyme is supported by the fact that dialysis failed to reactivate it. Reduction of $\mathrm{Fe}(\mathrm{CN})_{6}^{-3}$, which is carried out by the $E_{1}$ subunit, $\alpha$-ketoglutarate decarboxylase, was not inhibited by $\Delta 2,3 \mathrm{VPE}$, whereas $\mathrm{NAD}^{+}$reduction was inhibited. This observation suggests that binding of $\triangle 2,3$ VPE to KDHC is not at the $E_{1}$ subunit.

The mechanism of inactivation of KDHC by VPA-CoA and $\triangle 2,3$ VPE-CoA was investigated by testing the possibility that these compounds bind to the reduced $E_{2}$ subunit via a thioester bond, in a similar manner to its physiological product, succinyl-CoA:

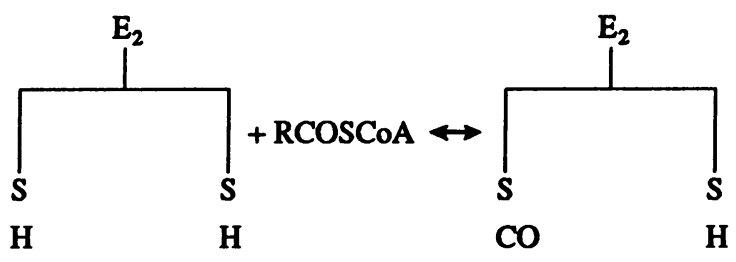

$\mathbf{R}$

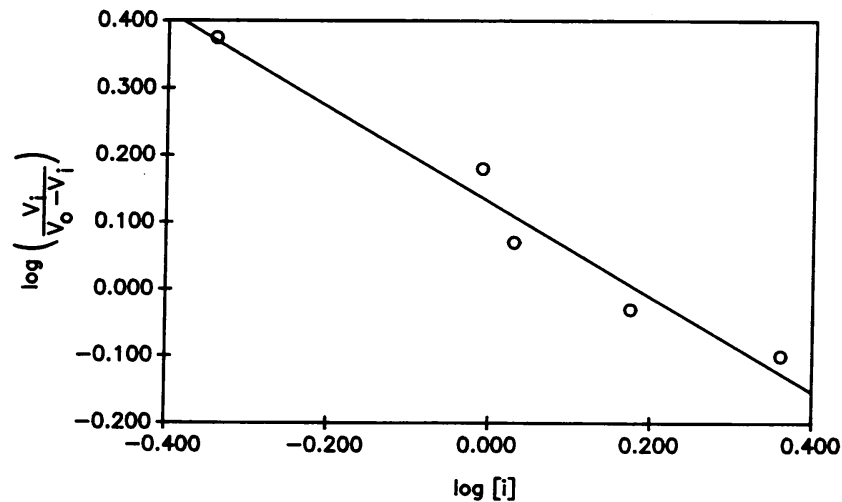

Figure 9 . Inhibition of purified beef brain $\alpha-\mathrm{KDHC}$ by $\delta 2,3 \mathrm{VPE}-$ CoA. Results are presented as a Hill plot. $V_{\mathrm{i}}$ is the initial rate of reaction in the presence of inhibitor and $V_{\mathrm{o}}$ the rate under the same conditions without inhibitor. CoA concentration was $1.3 \mu \mathrm{M}\left(=K_{\mathrm{m}}\right)$. Other substrates were as described in the text.
$E_{2}$ is the lipoamide succinyltransferase subunit of $\mathrm{KDHC}, \mathrm{R}$ is the acyl group, and $\mathrm{SH}$ the free sulfhydryl groups (after McMinn and Ottaway [33]). Excess free CoA can compete with the $E_{2}$-bound acyl group, if it is bound via a thioester bond, to regenerate the acyl-CoA and free sulfhydryl groups, in the reverse of reaction 1 . Succinyl-CoA is bound to $E_{2}$ relatively weakly and under physiologic conditions is displaced from $E_{2}$ by free CoA (33). It is proposed that the binding of VPA-CoA and $\triangle 2,3$ VPE-CoA is much tighter. The acyl groups would be displaced only slowly under physiological conditions in this model, but higher concentrations of CoA would shift the equilibrium in favor of displacement. The finding that re-activation of the enzyme occurred when excess free CoA $(0.5 \mathrm{mM})$ was added to the VPA-CoA or $\Delta 2,3$ VPECoA-inactivated enzyme is in support of this mechanism. The inactivation of KDHC by isobutyryl-CoA has been shown to occur by this mechanism (19). Further support for this is the fact that neither dialysis nor addition of $\mathrm{NAD}^{+}$nor $\alpha$-ketoglutarate, which do not have free sulfhydryl groups, were effective in reactivation. Binding of acyl-CoA by thioester bonds is dependent on the presence of reduced sulfhydryl groups. In this experiment NADH was present in order to reduce any oxidized lipoamide as follows:

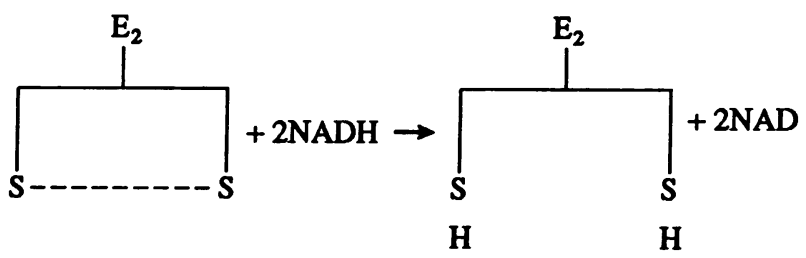

Table III. Inhibition of Beef Brain $\alpha-K D H C$ by Keto-acyl CoA Esters

\begin{tabular}{lcl}
\hline \multicolumn{1}{c}{ Inhibitor } & \multicolumn{1}{c}{$K_{\mathrm{is}}$} & Inhibition type \\
\hline & $\mu M$ & \\
Isovaleryl-CoA & $77.8 \pm 4.4$ & competitive \\
Tiglyl-CoA & $44.0 \pm 1.9$ & competitive \\
$n$-Propionyl-CoA & $49.2 \pm 1.8$ & competitive \\
Isobutyryl-CoA & $18.6 \pm 0.4\left(K_{\text {ii }}\right)$ & mixed \\
& $41.3 \pm 1.5\left(K_{\text {is }}\right)$ & \\
& &
\end{tabular}

Assays were carried out under steady state conditions and constants calculated as described in the legend to Table II. 


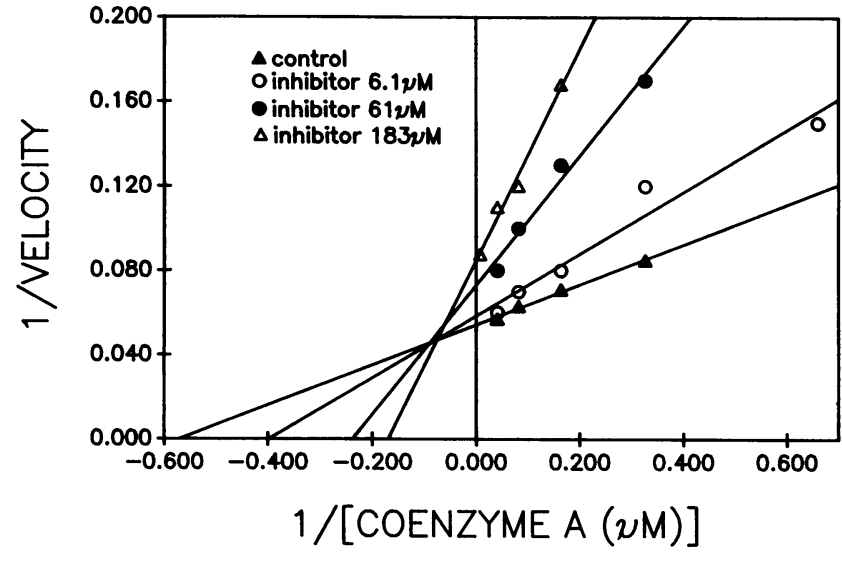

Figure 10. Inhibition of purified beef brain $\alpha-\mathrm{KDHC}$ by isobutyrylCoA. Assay conditions are as described in the legend of Fig. 7.

In addition to inactivation, we have also demonstrated that VPA metabolites and some naturally occurring fatty acylCoAs are potent (reversible) inhibitors of KDHC. Inhibition of $\mathrm{KDHC}$, which occurs in isolated rat hepatocytes under conditions favoring an increased acyl-CoA/free $\mathrm{CoA}$ ratio, is associated with reduced citric acid cycle flux (43-45). An increased acyl-CoA/free CoA ratio occurs in the presence of excess acyl groups, such as in patients with organic acidemias and other inborn errors of metabolism, and VPA therapy (46). Although our studies do not directly address the relative importance in vivo of inactivation and inhibition, the similarity of the kinetics of VPA metabolites and natural acyl-CoA esters present in excess in inborn errors supports the notion that inhibition of the citric acid cycle and KDHC by VPA may be a significant effect in vivo.

The work presented here provides a testable model for VPA action. Experiments to analyze GABA flux from the brain cell body and synaptosomic mitochondria treated with VPA are currently under way to study this model further.

\section{Acknowledgments}

The excellent technical assistance of Young-Hwa Lim is gratefully acknowledged. Dr. Paul Fennessy performed the mass spectrometry studies.

This work was supported by grants NS-24872 and NS-26520 from the National Institutes of Health to Dr. Parker, a Mental Retardation Research Center grant, and NIH Research Resource Grant RR-01152. Dr. Parker is the recipient of a Clinical Investigator Development Award from NINDS.

\section{References}

1. Meunier, H., G. Carray, Y. Meunier, P. Eymard, and M. Aicard. 1963. Pharmacology of 2-propyl valeric acid. Therapie (Paris). 18:435-483.

2. Zimmerman, H. J., and K. G. Ishak. 1982. Valproate-induced hepatic injury: analyses of 23 fatal cases. Hepatology (Baltimore). 2:591-597.

3. Simler, S., L. Chesielski, M. Maitre, H. Randrhanarisoa, and P. Mandel. 1973. Effects of sodium-n-dipropylacetate on audiogenic seizures and brain gamma-aminobutyric acid levels. Biochem. Pharmacol. 22:1701-1708.
4. Chapman, A., P. E. Keane, B. S. Meldrum, J. Simiand, and J. C. Vernieres. 1982. Mechanism of anticonvulsant action of valproate. Prog. Neurobiol. 19:315-359.

5. Taberner, P. B. 1979. Effects of sodium valproate and ethanolamine-o-sulphate on GABA metabolism. Br. J. Pharmacol. 67:441.

6. Turnbull, D. M., A. J. Bone, K. Barlett, P. P. Koundakjian, and H. S. A. Sheratt. 1983. The effects of valproate on intermediary metabolism in isolated rat hepatocytes and intact rats. Biochem. Pharmacol. 32:1887-1892.

7. Becker, C.-M., and R. A. Harris. 1983. Influence of valproic acid on hepatic carbohydrate and lipid metabolism. Arch. Biochem. Biophys. 223:381-392.

8. Eymard, P., M. Broll, and J. P. Werbend. 1970. Valproate diminishes oxygen utilization in rat hepatocytes. Bull. Soc. Sci. Vet. Med. Comp. Lyon. 72:303-325.

9. Kukino, K., and T. Deguchi. 1977. Effects of sodium dipropylacetate on GABA and biogenic amines in rat brain. Chem. Pharm. Bull. (Tokyo). 25:2257-2262.

10. Lai, J. C. K., and J. B. Clarke. 1979. Methods Enzymol. 55(Part F):51-60.

11. Williams, R. J. H., P. N. Patsalos, and R. Lowe. 1980. Changes in brain amino acid neurotransmitters induced by sodium valproate and their relevance to epilepsy and interactions with other anticonvulsants. In The Place of Sodium Valproate in the Treatment of Epilepsy. Royal Society of Medicine International Congress and Symposium No. 30. Academic Press, and The Royal Society, London. 95-102.

12. Haas, R., D. A. Stumpf, J. Parks, and L. Eguren. 1981. Inhibitory effects of sodium valproate on oxidative phosphorylation. Neurology. (NY). 31:1473-1476.

13. Cunningham, J., D. D. Clark, and W. J. Nicklas. 1980. Oxidative metabolism of 4-aminobutryrate by rat brain mitochondria: inhibition by branched chain fatty acid. J. Neurochem. 34:197-202.

14. Lai, J. C. K., and A. J. L. Cooper. 1986. Brain alpha-ketoglutarate dehydrogenase complex: kinetic properties, regional distribution and effects of inhibitors. J. Neurochem. 47:1376-1386.

15. Koike, M., and K. Koike. 1976. Structure, assembly and function of mammalian alpha-keto acid dehydrogenase complexes. $A d v$ Biophys. 9:187-227.

16. Ito, M., Y. Ikeda, G. Finocchiaro, J. Arnez, and K. Tanaka. 1986. The enzymatic basis for the metabolism and inhibitory action of valproate: dehydrogenation of VPA-CoA by 2-methyl-branched chain acyl-CoA dehydrogenase. Pediatr. Res. 20:343A. (Abstr.)

17. Nau, H., and W. Loescher. 1982. Valproic acid: brain and plasma levels of the drug and its metabolites, anticonvulsant effects and GABA metabolism in the mouse. J. Pharmacol. Exp. Ther. 220:654-659.

18. Loescher, W., H. Nau, and M. Marescaux-Vergnes. 1984. Comparative evaluation of anticonvulsant and toxic potencies of valproic acid and 2-en-valproic acid in different animal models of epilepsy. Eur. J. Pharmacol. 99:211-218.

19. Jackson, R. H., and T. P. Singer. 1983. Inactivation of the 2-ketoglutarate and pyruvate dehydrogenase complexes of the beef heart by branched chain keto acids. J. Biol. Chem. 258:1857-1865.

20. Wilkinson, G. N. 1961. Statistical estimations in enzyme kinetics. Biochem. J. 80:324-333.

21. Lowry, O. H., N. J. Rosebrough, A. L. Farr, and R. J. Radials. 1951. Protein measurement with the folin phenol reagent. J. Biol. Chem. 193:265-275.

22. Laemmli, U. K. 1970. Cleavage of structural proteins during the assembly of the head of bacteriophage T4. Nature (Lond.). 227:680-685.

23. Perham, R. N., and J. O. Thomas. 1971. The subunit molecular weights of the $\alpha$-keto acid dehydrogenase multienzyme complexes from E. coli. FEBS (Fed. Eur. Biochem. Soc.) Lett. 15:8-12.

24. Barrera, C. R., G. Namihara, L. Hamilton, P. Munk, M. H. Eley, T. C. Linn, and L. J. Reed. 1972. Alpha-ketoacid dehydrogenase complexes-XVI. Arch. Biochem. Biophys. 148:343-347.

25. Weber, K., and M. Osborn. 1969. The reliability of molecular 
weight determinations by dodecyl sulfate-polyacrylamide gel electrophoresis. J. Biol. Chem. 244:4406-4410.

26. Tanaka, N., K. Koike, K.-I. Otsuka, M. Hamada, K. Ogashahara, and M. Koike. 1972. Mammalian $\alpha$-ketoacid dehydrogenase complexes. J. Biol. Chem. 249:191-198.

27. Bernert, J. T., and H. Sprecher. 1977. An analysis of partial reactions in the overall chain elongation of saturated and unsaturated fatty acids by rat liver microsomes. J. Biol. Chem. 252:6736-6744.

28. Causey, A. G., B. Middleton, and K. Barlett. 1986. A study of the metabolism of $\left[\mathrm{U}-{ }^{14} \mathrm{C}\right] 3$-methyl-2-oxopentanoate by rat liver mitochondria using h.p.l.c. with continuous on-line monitoring of radioactive intact acyl-coenzyme A intermediates. Biochem. J. 235:343350.

29. Myers, D. E., and M. F. Utter. 1981. The enzymatic synthesis of some potential photoaffinity analogs of benzoyl-coenzyme A. Anal. Biochem. 112:23-29.

30. Kanzaki, T., T. Hayakawa, M. Hamada, Y. Fukuyoshi, and M. Koike. 1969. Mammalian $\alpha$-ketoacid dehydrogenase complexes-IV. J. Biol. Chem. 244:1183-1187.

31. Parker, P. J., and P. J. Randle. 1978. Partial purification and properties of branched chain 2-oxo acid dehydrogenase of ox liver. Biochem. J. 171:751-757.

32. Smith, C. M., J. Bryla, and J. R. Williamson. 1974. Regulatoin of mitochondrial alpha-ketoglutarate metabolism by product inhibition at alpha-ketoglutarate dehydrogenase. J. Biol. Chem. 249:14971505.

33. McMinn, C. L., and J. H. Ottaway. 1977. Studies on the mechanism and kinetics of the 2-oxoglutarate dehydrogenase system from pig heart. Biochem. J. 161:569-581.

34. Hirashima, M., T. Hayakawa, and M. Koike. 1967. Mammalian $\alpha$-ketoacid dehydrogenase complexes-II. J. Biol. Chem. 242:902-907.

35. Massey, V. 1960. The composition of the ketoglutarate dehydrogenase complex. Biochem. Biophys. Acta. 38:447-460.
36. Ishikawa, E., R. M. Oliver, and L. J. Reed. 1966. Alpha-ketoacid dehydrogenase complexes, V. Proc. Natl. Acad. Sci. USA. 56:534-541.

37. Martin-Requero, A., B. E. Corkey, S. Cerdan, E. WalajtysRode, R. L. Parrilla, and J. R. Williamson. 1983. Interactions between $\alpha$-ketoglutarate metabolism and the pathways of gluconeogenesis and urea synthesis in isolated hepatocytes. J. Biol. Chem. 258:3673-3681.

38. Löscher, W., and H. Nau. 1983. Distribution of valproic acid and its metabolites in various brain areas of dogs and rats after acute and prolonged treatment. J. Pharmacol. Exp. Ther. 226:845-854.

39. Aly, M. I., and A. A. Abdel-Latif. 1980. Studies on the distribution and metabolism of valproate in rat brain, liver and kidney. Neurochem. Res. 5:1231-1242.

40. Harding, G. F. A., C. E. Herrick, and P. M. Jeavons. 1978. A controlled study of the effect of sodium valproate on photosensitive epilepsy and its prognosis. Epilepsia. 19:555-565.

41. Johnston, D., and G. E. Slater. 1982. Valproate-mechanisms of action. In Antiepileptic Drugs. D. M. Woodbury, J. K. Penry, and C. E. Pippenger, editors. Raven Press, New York. 611-616.

42. Lockard, J. S., and R. H. Levy. 1976. Valproic acid: reversibly acting drug? Epilepsia. 17:477-479.

43. Williamson, J. R., E. Walajtys-Rode, and K. E. Coll. 1979. Effects of branch chain alpha-ketoacids on the metabolism of isolated rat liver cells-1. J. Biol. Chem. 254:11511-11520.

44. Walajtys-Rode, E., K. E. Coll, and J. R. Williamson. 1979. Effects of branch chain alpha-ketoacids on the metabolism of isolated rat liver cells-2. J. Biol. Chem. 254:11521-11529.

45. Walajtys-Rode, E., and J. R. Williamson. 1980. Effects of branch chain alpha-ketoacids on the metabolism of isolated rat liver cells-3. J. Biol. Chem. 255:413-418.

46. Turnbull, D. M., D. J. Dick, L. Wilson, H. S. A. Sheratt, and K. G. M. M. Alberti. 1986. Valproate causes metabolic disturbance in normal man. J. Neurol. Neurosurg. Psychiatry. 49:405-410. 